\title{
Peculiarities of the Ionic Dynamics of Molten Nitrites
}

\author{
S. A. Kirillov \\ Institute of General and Inorganic Chemistry (Ukrainian S.S.R. Academy of Sciences), Kiev, USSR \\ D. Tunega \\ Institute of Inorganic Chemistry, Centre for Chemical Research (Slovak Academy of Sciences), \\ Bratislava, Czechoslovakia
}

Z. Naturforsch. 45a, 145-147 (1990); received September 27, 1989

Polarization measurements in the Raman spectra of molten sodium nitrite have shown that the reorientational line widths of the $v_{1}$ and $v_{2}$ bands (both of the $A_{1}$ symmetry type) of $\mathrm{NO}_{2}^{-}$are different. Possible reasons are discussed. Also, the line widths of $\mathrm{NO}_{2}^{-1}$ for various molten nitrites are found to depend on $R^{-4}$, the cloasest approach distance in the melt, and on $Z$, the cation charge number. This dependence agrees with theoretical predictions for the coupling of vibrational and reorientational dephasing caused by ion-dipole interactions.

\section{Introduction}

The usual assumption that for a given vibrational mode the vibrational correlation functions in infrared, isotropic and anisotropic Raman are equal was shown to be invalid in two kinds of experiments. When studying different Raman bands of the same symmetry type for the same molecule one may find the reorientational part of line widths to vary from band to band, although reorientation about the same molecular axis is probed [1]. Differences of the vibrational parts of infrared and Raman line widths for the same normal mode can also exist [2]. This phenomenon was discussed in several papers (see, e.g., [3-6]).

Such a phenomenon appears to be unknown for molten salts, the systems involving strong interparticle interactions. Nevertheless, the examination of recent spectroscopic data proves its existence for molten alkali nitrates. Derivation of $\tau_{1 \mathrm{R}}$ and $\tau_{2 \mathrm{R}}$ correlation times from IR [7] and Raman [8] spectra using $\tau_{\mathbf{v}}$ from isotropic Raman band contours for the same $v_{1}\left(A_{1}^{\prime}\right)$ normal mode of $\mathrm{NO}_{3}^{-}$gives erroneous results: $\tau_{1 \mathrm{R}} \ll \tau_{2 \mathrm{R}}$ for all salts studied excepting $\mathrm{CsNO}_{3}$. The aim of this work is to study the differences of the reorientational line width probing the different $v_{1}$ and $v_{2}$ bands of the same $A_{1}$ symmetry type for the same entity, $\mathrm{NO}_{2}^{-}$, in a single Raman experiment.

Reprint requests to Doc. Dr. S. A. Kirillov, Institute of General and Inorganic Chemistry, 32-34 Prospect Palladina, 252601 Kiev-142, USSR.

\section{Experimental}

Owing to the instability of molten nitrites, Raman spectra of sufficiently high quality were obtained for sodium nitrite only. The chemically pure (USSR) salt under investigation was first recrystallized, its hot solution was filtered and dried. Raman spectra were collected on a JEOL JRS-S1 spectrometer equipped with the RS-HT2 high temperature Raman cell device. The $514.5 \mathrm{~nm}$ line of an argon ion laser (ILA 120) was used at an incident power output of $150 \mathrm{~mW}$ at the sample. For scattered radiation with different polarization vector orientations, the isotropic and anisotropic scattering components were isolated according to the relations

$$
\begin{aligned}
& I_{\text {aniso }}(v)=I_{\perp}(v), \\
& I_{\text {iso }}(v)=I_{\|}(v)-\frac{4}{3} I_{\perp}(v) .
\end{aligned}
$$

The depolarization ratio of the nitrite ion $v_{2}$ line was 0.050 . The tentative depolarization ratio of the $v_{1}$ line was 0.24 (0.31 according to [9]). A more accurate estimation is difficult since this polarized line and the depolarized line $v_{3}(E)$ overlap on the small wave number side. This circumstance does not allow the whole $I_{\text {aniso }}(v)$ contour ot be found with good accuracy and forces to use the overlap-free side only. The spectra are shown in Fig. 1 and the line widths are summarized in Table 1. Clearly the reorientational line widths $\delta_{2 \mathrm{R}}=\delta_{\text {aniso }}-\delta_{\text {iso }}$ from $v_{1}$ and $v_{2}$ are different. 

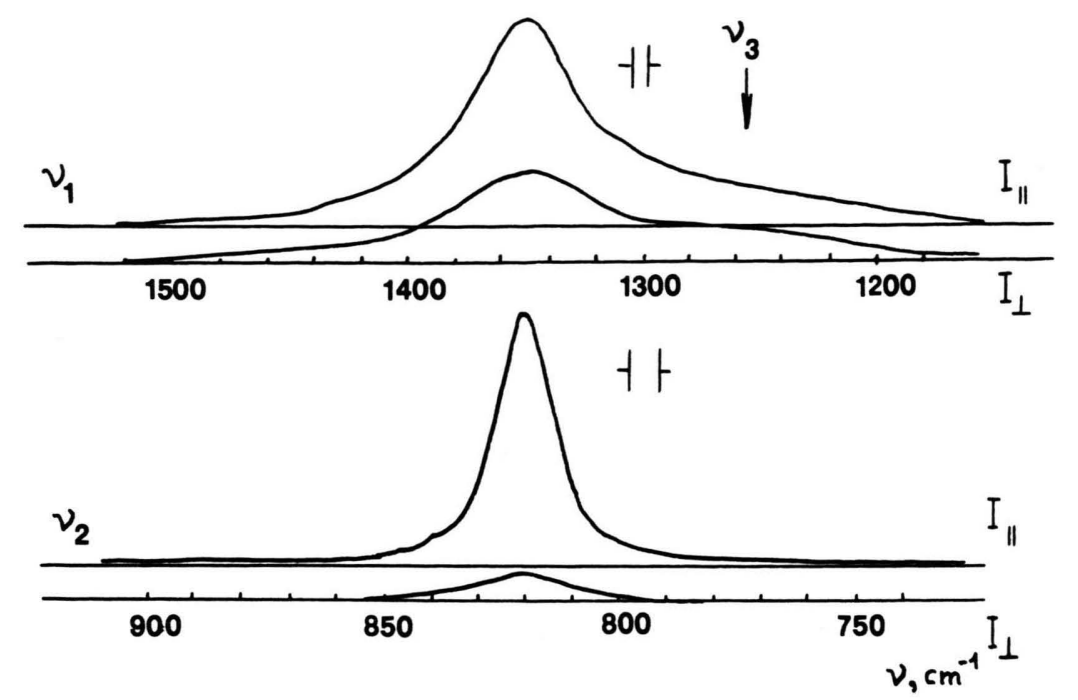

Fig. 1. Raman spectra of $\mathrm{NaNO}_{2}$ at $573 \mathrm{~K}$.

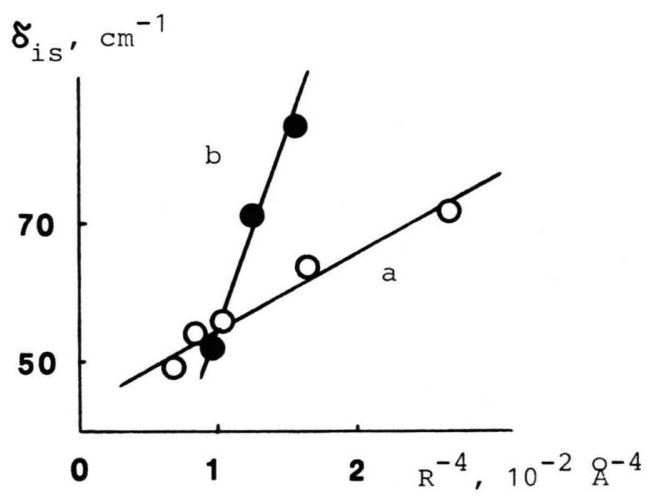

Fig. 2. Raman line widths of the $v_{1}$ mode of $\mathrm{NO}_{2}^{-}$ion in molten alkali (a) and alkaline earth (b) nitrites plotted against $R^{-4}$. All data taken from [9], cationic radii according Batsanov [14], $R_{\mathrm{NO}_{\overline{2}}^{-}}=1.8 \AA$ [15].

\section{Discussion}

According to Lynden-Bell, the difference between the vibrational contributions to the infrared, isotropic and anisotropic Raman line contours can arise either from the existence of cross terms between the "self" and "distinct" parts of the vibrational correlation function [3] or from the coupling of vibrational and reorientational dephasing [4]. The former appears to be of no importance for melts. Expanding the interparticle potential $F$ in a Taylor series with respect to normal coordinates $Q$, Lynden-Bell distinguishes between two terms which contribute to the secular broadening: The diagonal term, $\frac{1}{2} \frac{\partial^{2} F}{\partial Q_{\mathrm{i}}^{2}} Q_{\mathrm{i}}^{2}$, and
Table 1. Line widths (full widths at half hights in $\mathrm{cm}^{-1}$ ) in the Raman spectra of molten $\mathrm{NaNO}_{2} . \partial \mu / \partial Q_{\mathrm{i}}$ were calculated in arbitrary units using the IR intensity data for solid solutions of nitrites in $\mathrm{KCl}[10]$.

\begin{tabular}{lllll}
\hline Mode & $\delta_{\text {iso }}$ & $\delta_{\text {aniso }}$ & $\delta_{2 \mathrm{R}}$ & $\frac{\partial \boldsymbol{\mu}}{\partial Q_{\mathrm{i}}}$ \\
\hline$v_{1}$ & 54.4 & 80.0 & 25.6 & 1.9 \\
$v_{2}$ & 14.8 & 21.2 & 6.4 & 1 \\
\hline
\end{tabular}

the off diagonal exchange (resonant transfer) term, $\frac{1}{2} \frac{\partial^{2} F}{\partial Q_{\mathrm{i}} \partial Q_{\mathrm{j}}} Q_{\mathrm{i}} Q_{\mathrm{j}}$, where $\mathrm{i}$ and $\mathrm{j}$ label the particle of interest and its surroundings. If the interionic potential is divided, following Hills and Madden [11], into cation-anion and anion-anion parts, one may conclude that the former gives no contribution to the exchange term. However, the contribution of anionanion interaction is negligibly small for melts since anions are surrounded by cationic layers. As follows from recent reviews $[12,13]$, resonant transfer is not actually the cause of line shifts and broadening in melts even for the lines which are most intense in infrared. If the vibration is anharmonic there exists another self term which is proportional to the first derivative of the interaction potential: $\left(\partial F / \partial Q_{\mathrm{i}}\right)_{0} Q_{\mathrm{i}}$. In real cases it can be of the same order of magnitude as the second derivative term.

If one follows Lynden-Bells theory of coupling of vibrational and reorientational dephasing $[4,5]$, the magnitude of such coupling may be measured with the square of the orientationally dependent contribution 
of the interionic potential. Therefore one can expect a strong coupling in the case of the ion-dipole contribution. The first and second derivative diagonal terms are then proportional to

$$
\left(\frac{\partial^{n} \mu_{\mathrm{i}}}{\partial Q_{\mathrm{i}}^{n}}\right)_{0} \frac{Z e}{R^{2}} Q_{\mathrm{i}}^{n} \quad \text { with } \quad n=1 \text { or } 2,
$$

where $\mu_{\mathrm{i}}$ is the anion dipole moment, $Z$ the cation charge number, $e$ the electron charge and $R$ the distance of closest approach. The slope of $\delta_{\text {iso }}$ against $R^{-4}$ for divalent cations is actually almost four times as large as for univalent ones (Figure 2). This observation confirms the assumption that the isotropic width is mainly due to ion-dipole interaction, because in the fast modulation limit the width is proportional to the

[1] A. M. Amorim da Costa, M. A. Norman, and J. H. R. Clarke, Molec. Phys. 29, 191 (1975).

[2] J. Yarwood, P. L. James, G. Döge, and R. Arndt, Faraday Disc. Chem. Soc. 66, 252 (1978).

[3] S. Bratos and G. Tarjus, Can. J. Chem. 63, 2047 (1985).

[4] R. M. Lynden-Bell, Molec. Phys. 33, 907 (1977).

[5] R. M. Lynden-Bell, Molec. Phys. 36, 1529 (1978).

[6] P. C. M. van Woerkom, J. de Bleijser, M. de Zwart, and J. C. Leyte, Chem. Phys. 4, 236 (1974).

[7] S. A. Kirillov and A. V. Gorodyskii, Dokl. Acad. Nauk. SSSR 265, 906 (1972).

[8] T. Kato and T. Takenaka, Mol. Phys. 54, 1393 (1985).

[9] V. D. Prisyazhnyi, S. A. Kirillov, and V. I. Snezhkov, Zhurn. Prikl. Spektr. 25, 1058 (1976). 2nd moment, the averaged square of the spectroscopically active interionic potential. The fact that the larger $\left(\partial \mu / \partial Q_{\mathrm{i}}\right)_{0}$, the broader the isotropic part of the bands studied (Table 1) could indicate at a first sight that the first derivative term of the interaction potential is more important in these cases than the second derivative term. Indeed that is true for $\mathrm{NO}_{2}$-because the first overtone of the $v_{1}$ mode is undetectable in IR (see [10] and references cited therein).

\section{Acknowledgement}

One of us (S.A.K.) is very grateful to Doc. Dr. K. Matiašovský and Dr. M. Pisárčik for their kind hospitality during his stay in Bratislava.

[10] R. Bonn, R. Metselaar, and J. van der Elsken, J. Chem. Phys. 46, 1988 (1967).

[11] B. P. Hills and P. A. Madden, J. Chem. Soc. Faraday Trans. II, 75, 220 (1979)

[12] M. H. Brooker and G. N. Papatheodorou, in: Adv. Molten Salt Chem., vol. 5 (G. A. Mamantov et al., ed.), Plenum, New York 1985, p. 26.

[13] S. A. Kirillov, in: Dynamic Properties of Molecules and Condenced Media (A. N. Lazarev, ed.) (in Russian), Nauka, Leningrad 1988, p. 190.

[14] S. S. Batsanov, in: Short Chem. Encyclopaedia (in Russian), Sov. Encyclopaedia, Moscow 1963, vol. 2, p. 310.

[15] Y. Iwadate, K. Kawamura, K. Igarashi, and J. Mochinaga, J. Phys. Chem. 86, 5205 (1982). 